\title{
A STOCHASTIC ORDERING PROPERTY FOR LEAKY BUCKET REGULATED FLOWS IN PACKET NETWORKS
}

\author{
FABRICE M. GUILLEMIN, ${ }^{*}$ France Telecom \\ RAVI R. MAZUMDAR ${ }^{* * * * *}$ AND \\ CATHERINE P. ROSENBERG, ${ }^{* * * * * *}$ University of Waterloo \\ YU YING, ${ }^{* * * * *}$ Purdue University
}

\begin{abstract}
We show in this paper that if a stationary traffic source is regulated by a leaky bucket with leak rate $\rho$ and bucket size $\sigma$, then the amount of information generated in successive time intervals is dominated, in the increasing convex ordering sense, by that of a Poisson arrival process with rate $\rho / \sigma$, with each arrival bringing an amount of information equal to $\sigma$. By exploiting this property, we then show that the mean value in the stationary regime of the content of a buffer drained at constant rate and fed with the superposition of regulated flows is less than the mean value of the same buffer fed with an adequate Poisson process, whose characteristics depend upon the regulated input flows.
\end{abstract}

Keywords: Stochastic ordering; fluid queue; regulated flows

2000 Mathematics Subject Classification: Primary 60K35

Secondary 90B15; 90B18; 90B20

\section{Introduction}

The leaky bucket is an algorithm that was introduced in [15] in the mid 1980s in order to control the bit rate of a traffic source at the access point of a packet network. The algorithm is characterized by two parameters: the leak rate $\rho$, which is the long run achievable bit rate, and a bucket size $b$, which allows for fluctuations around the average bit rate $\rho$. In addition to these two parameters and related to the mean bit rate is the maximum transmission rate $\pi>\rho$ of the source, which is limited by a second leaky bucket with a bucket size set equal to 0 . The basic principle of the leaky bucket is as follows: the algorithm maintains a bucket counter expressed in bits. When a packet of length $P$ bits arrives at the leaky bucket, the following procedure is invoked:

- if the counter value is greater than $P$, then the packet is admitted into the network and the counter value is decreased by $P$ bits;

Received 20 December 2005; revision received 27 March 2007.

* Postal address: France Telecom, 2 Avenue Pierre Marzin, 22300 Lannion, France.

Email address: fabrice.guillemin@ orange-ftgroup.com

** Postal address: Department of Electrical and Computer Engineering, University of Waterloo, Waterloo, ON N2L 3G1, Canada.

*** Email address: mazum@ece.uwaterloo.ca

**** Email address: cath@ece.uwaterloo.ca

***** Postal address: School of ECE, Purdue University, West Lafayette, IN 47907, USA.

Email address: yingy@purdue.edu 
- if the counter value is less than $P$, then the packet is discarded and the counter value is left unchanged.

As a background task, the counter value is continually incremented at rate $\rho$ (in bit/s) as long as it does not exceed the maximum value $b$.

For modeling purposes, it is often more convenient to consider fluid flow approximations. In that case, traffic sources are assumed to transmit bits instead of packets. Bits are supposed to be infinitely small and are admitted by the leaky bucket as long as the bucket counter value is positive. Because of the bucket size $b$, a traffic source can transmit bursts at the peak rate with size $\sigma=b \pi /(\pi-\rho)$. In fact, because of the dual leaky bucket control, the quantity of information $A(s, t]$, which can be admitted into the network in any time interval $(s, t]$ must satisfy

$$
A(s, t] \leq \min (\pi(t-s), \sigma+\rho(t-s)) .
$$

The peak rate constraint is often implicit and omitted in the above inequality. In practical situations, the peak rate is set equal to a typical link rate (e.g. $155 \mathrm{Mbit} / \mathrm{s}$ for an OC3 access link rate to an asynchronous transfer mode network or 1Gbit/s for an ethernet access link to an IP backbone network). A flow satisfying the constraint

$$
A(s, t] \leq \sigma+\rho(t-s),
$$

is said to be $(\sigma, \rho)$-regulated.

The study of $(\sigma, \rho)$-regulated flows has been central in the development of packet networks since the beginning of the 1990s. In two seminal papers, Cruz [5], [6] showed that it is possible to derive an upper bound for the delay experienced by a $(\sigma, \rho)$-regulated flow through the network offering minimum bandwidth guarantees. These papers laid down the basis of a new research area in networking, referred to as network calculus. A new formalism based on $(\mathrm{min},+)$ algebra was developed first by [3] and then by [12] in order to manipulate and derive bounds on delays for $(\sigma, \rho)$-regulated flows for different service disciplines and admission policies in network elements.

A major shortcoming of the deterministic approach based on (min, + ) algebra, however, is that delay bounds are very loose and do not account for randomness, when multiplexing several regulated flows. In fact, obtaining accurate bounds when multiplexing regulated flows in a buffer is a recurrent open problem in the framework of network calculus. Several approaches have been proposed in the literature to obtain an upper bound for $\mathrm{P}(w>x)$, where $w$ is the content of a buffer fed with one or several regulated flows and drained at a constant rate $c$, and $x$ is an arbitrary buffer level. For instance, when sources are homogeneous (i.e. characterized by the same triplet, $(\sigma, \rho, \pi)$, as described above), bounds have been obtained by [8], [11], [4], etc. An overview of the different techniques as well as an exhaustive bibliography on the subject can be found in [7]. It is also worth noting that [14] obtained a stochastic bound (in the increasing convex ordering sense; see [1, Chapter 4] and definitions in Section 2 for details on stochastic ordering) for the quantity $A(0, t]-\rho t$ in the form

$$
A(0, t]-\rho t \leq_{\text {icx }} X_{\sigma},
$$

where the random variable $X_{\sigma}$ is defined by $\mathrm{P}\left(X_{\sigma}= \pm \sigma\right)=\frac{1}{2}$. The major advantage of this latter bound is that it is intrinsic to a regulated traffic source without referring to the stationary characteristics of a buffer (e.g. the buffer content in the stationary regime).

In this paper we follow the same line of investigations as in [9] and [10], where the content of a buffer fed with the superposition of regulated flows and drained at a constant rate $c$ is 
compared with the content of the same buffer fed with a batch Poisson process. We specifically investigate the 'better than Poisson' property, which amounts to stating that the content $w$ of a buffer drained at a constant rate and fed with the superposition of regulated flows is less than or equal to the content $W$ of the same buffer fed with an appropriate Poisson process. In general, 'better than Poisson' is understood in the strong ordering sense (i.e. in the notation of [1], $w \leq_{i} W$ ). Only results for the asymptotic behavior of the above queues can, however, be rigorously proved (see [13] for instance). In this paper, we obtain a finer result by showing that a $(\sigma, \rho)$-regulated flow is 'better than Poisson' in the increasing convex ordering sense. Using the same notation as above, this entails that the random variable $w$ is in the increasing convex ordering sense less than or equal to $W$.

The organization of this paper is as follows: In Section 2, we introduce the notation and the definitions used in the subsequent sections. In Section 3, we prove the 'better than Poisson' property of a $(\sigma, \rho)$-regulated flow. In Section 4 , we investigate the queueing implications of this property.

\section{Problem formulation}

Consider a stochastic $(\sigma, \rho)$-regulated source defined on some reference filtered probability space $\left(\Omega, \mathcal{F}, \mathrm{P},\left(\mathcal{F}_{t}\right)\right)$. This means that the process describing the quantity of information, which can be transmitted by the source in an arbitrary time interval $(s, t]$ and which is a random variable denoted by $A(s, t]$, must satisfy, for almost every sample path $\omega \in \Omega$,

$$
A(s, t](\omega) \leq \sigma+\rho(t-s),
$$

where $\sigma$ and $\rho$ are positive real numbers. To simplify the notation, we set

$$
A(t)= \begin{cases}A(0, t] & \text { if } t \geq 0 \\ A(t, 0] & \text { if } t \leq 0\end{cases}
$$

In addition, we assume that the source is stationary in the sense that the process $(A(t))$ has stationary increments. The process $(A(t))$ is referred to as the arrival process of the $(\sigma, \rho)$-regulated source and is supposed to be continuous from the right with left limits (càdlàg).

Sources satisfying the $(\sigma, \rho)$-constraint $(2.1)$ can transmit bursts, which correspond to amounts of information arriving at once (i.e. in time intervals of length 0 ). These bursts appear as jumps in the arrival process of the source. The peak rate constraint mentioned in the introduction then applies to the right derivative of the process $(A(t))$. Because of the $(\sigma, \rho)$-constraint, the maximal size of a burst is equal to $\sigma$ and the distance between two consecutive bursts of size $\sigma$ is greater than or equal to $\sigma / \rho$. In addition, if a $(\sigma, \rho)$-regulated source is stationary, the point process counting jumps in the arrival process $A(t)$, if any, is a stationary point process. In this case, the source is said to be with bursts. In the case when there are no bursts, the arrival process $A(t)$ is continuous.

Let us fix some time constant $\tau>0$. We denote by $\left(A^{\tau}(t)\right)$ the arrival process that is periodic and transmitting bursts of size $\sigma$, followed by an activity period of length $\tau$ at rate $\rho$ and then by a silence period of length $\sigma / \rho$ before resuming a new activity period. The time origin being fixed, we assume that the phase of this periodic source is arbitrary, that is, the distance between the time origin and the instant of the first burst of size $\sigma$ is uniformly distributed in the time interval $(0, \tau+\sigma / \rho)$. A sample path of the arrival process $\left(A^{\tau}(t)\right)$ is depicted in Figure 1 . 


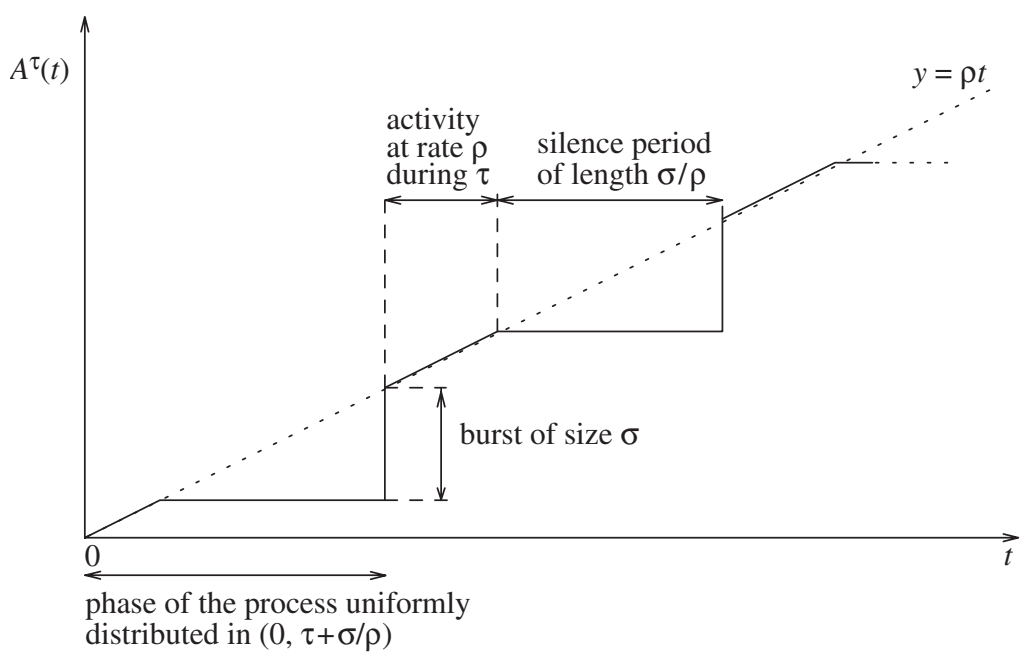

FIGURE 1: Sample path of the arrival process $\left(A^{\tau}(t)\right)$.

If $\rho \tau \leq \sigma$, straightforward computations show that

$$
A^{\tau}(\tau)= \begin{cases}0 & \text { with probability }(\sigma / \rho-\tau) /(\tau+\sigma / \rho), \\ \rho(\tau-u) & \text { with PDF } \mathbf{1}_{\{u \leq \tau\}} \mathrm{d} u /(\tau+\sigma / \rho), \\ \sigma+\rho\left(u-\frac{\sigma}{\rho}\right) & \text { with PDF } \mathbf{1}_{\{\sigma / \rho \leq u \leq \tau+\sigma / \rho\}} \mathrm{d} u /(\tau+\sigma / \rho),\end{cases}
$$

where PDF stands for the probability density function. Then, for $x \geq 0$,

$$
\mathrm{P}\left(A^{\tau}(\tau)>x\right)= \begin{cases}\frac{2 \rho \tau-x}{\sigma+\rho \tau} & \text { for } x \leq \rho \tau, \\ \frac{\rho \tau}{\sigma+\rho \tau} & \text { for } \rho \tau \leq x \leq \sigma, \\ \frac{\sigma+\rho \tau-x}{\sigma+\rho \tau} & \text { for } \sigma \leq x \leq \sigma+\rho \tau, \\ 0 & \text { for } x \geq \sigma+\rho \tau .\end{cases}
$$

Note that if $\sigma>\rho \tau, \mathrm{P}\left(A^{\tau}(\tau)=0\right)=(\sigma-\rho \tau) /(\sigma+\rho \tau)$.

If $\rho \tau \geq \sigma$,

$$
A^{\tau}(\tau)= \begin{cases}\rho(\tau-u) & \text { with PDF } \mathbf{1}_{\{u \leq \sigma / \rho\}} \mathrm{d} u /(\tau+\sigma / \rho), \\ \rho \tau & \text { with probability }(\tau-\sigma / \rho) /(\tau+\sigma / \rho), \\ \rho u & \text { with PDF } \mathbf{1}_{\{\tau \leq u \leq \tau+\sigma / \rho\}} \mathrm{d} u /(\tau+\sigma / \rho),\end{cases}
$$


then

$$
\mathrm{P}\left(A^{\tau}(\tau)>x\right)= \begin{cases}1 & \text { for } x \leq \rho \tau-\sigma, \\ \frac{2 \rho \tau-x}{\sigma+\rho \tau} & \text { for } \rho \tau-\sigma \leq x \leq \rho \tau, \\ \frac{\sigma+\rho \tau-x}{\sigma+\rho \tau} & \text { for } \rho \tau \leq x \leq \sigma+\rho \tau, \\ 0 & \text { for } x \geq \sigma+\rho \tau .\end{cases}
$$

Note that if $\sigma<\rho \tau$, the random variable $A^{\tau}(\tau)$ has a mass at point $\rho \tau$ with magnitude $(\rho \tau-\sigma) /(\sigma+\rho \tau)$.

The goal of this paper is to compare, in the sense of strong and increasing convex orderings (see [1] for details), the random variable $A(\tau)$ with $A^{\tau}(\tau)$ and $B(\tau)$, which has a Poisson distribution on the set $\{k \sigma, k \geq 0\}$. In addition, we extend the strong and increasing convex orderings for random variables to stochastic processes as follows. Let $\mathcal{L}$ be a stochastic ordering. We shall say in the rest of this paper that an arrival process $(A(t))$ is dominated for the stochastic ordering $\mathcal{L}$ by an arrival process $(B(t))$, denoted by $(A(t)) \leq_{\mathscr{L}}(B(t))$, if for every positive integer $n$ and arbitrary $t_{1}<t_{2}<\cdots<t_{n}$ in $\mathbb{R}$,

$$
\left(A\left(t_{1}\right), A\left(t_{2}\right), \ldots, A\left(t_{n}\right)\right) \leq_{\mathcal{L}}\left(B\left(t_{1}\right), B\left(t_{2}\right), \ldots, B\left(t_{n}\right)\right) .
$$

\section{3. 'Better than Poisson' property for regulated flows}

In this section we show that a $(\sigma, \rho)$-regulated arrival process is dominated, in the increasing convex ordering sense, by an appropriate batch Poisson process, which is defined as follows.

Definition 3.1. (Batch Poisson process.) A batch Poisson process with rate $\lambda$ and batch size $\sigma$ is a Poisson process with rate $\lambda$ such that with each point is associated a quantity of information equal to $\sigma$. The random quantity of information generated by such a batch Poisson process in a time interval of length $t$ takes the value $k \sigma$ with the probability $(\lambda t)^{k} \mathrm{e}^{-\lambda t} / k$ ! for nonnegative integer $k$.

In the following, we consider a $(\sigma, \rho)$-regulated arrival process $(A(t))$ and a batch Poisson process $(B(t))$ with arrival rate $\rho / \sigma$ and batch size $\sigma$. As a first step, we intend to show the following result.

Proposition 3.1. If $(A(t))$ is a $(\sigma, \rho)$-regulated arrival process and $(B(t))$ is a batch Poisson process with arrival rate $\rho / \sigma$ and batch size $\sigma$, then, for any $\tau>0, A(\tau) \leq_{\text {icx }} B(\tau)$.

To prove the above result, we establish a series of technical lemmas. First, we consider the case of an arrival process with bursts of size $\sigma$.

Lemma 3.1. Consider an arbitrary stationary $(\sigma, \rho)$-regulated arrival process $(A(t))$ with jumps of size $\sigma$ and fix some time constant $\tau>0$. Then, $A(\tau) \leq_{s t} A^{\tau}(\tau)$, where $\left(A^{\tau}(t)\right)$ is the periodic arrival process with bursts separated by a time period of length $\tau+\sigma / \rho$, a burst being followed by an activity period of duration $\tau$, followed in turn by a silence period of length $\sigma / \rho$.

Proof. Let us first consider the case $\rho \tau \leq \sigma$. Let $(N(t))$ denote the point process counting the jumps of size $\sigma$. The points of $(N(t))$ are denoted by $\cdots<T_{0} \leq 0<T_{1}<T_{2}<\cdots$. Owing to the $(\sigma, \rho)$-constraint (2.1), we have $T_{n}-T_{n-1}>\sigma / \rho$, for all $n$. As the source is assumed to be stationary, the point process $(N(t))$ is stationary. 


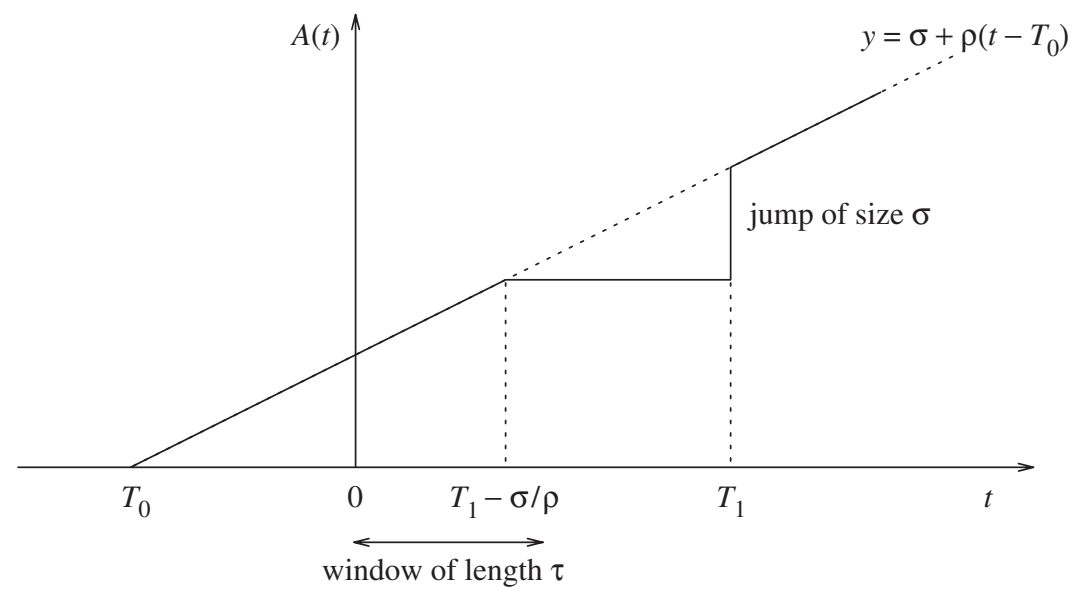

FigURE 2: Constraints for the arrival process $(A(t))$ in a time interval of length $\tau$.

We search for a traffic profile, which is maximal in the strong ordering sense, i.e. so that $\mathrm{P}(A(\tau)>x)$ is maximal for all $x \in(0, \sigma+\rho \tau)$. The point zero falls at random into the interval $\left[T_{0}, T_{1}\right]$. If $F$ denotes the probability distribution function of $\left(T_{1}-T_{0}\right)$, then the distribution of $T_{1}$ has the survival probability density function $(1-F(x)) / \mathrm{E}\left(T_{1}-T_{0}\right)$. Because of the $(\sigma, \rho)$-constraint, for any realization $\omega$, the curve $t \rightarrow A(t)$ is dominated by the curve $t \rightarrow \min \left(\rho\left(t-T_{0}\right), \rho\left(T_{1}-T_{0}\right)-\sigma\right)$. Indeed, to allow for a jump of size $\sigma$ at time $T_{1}$, the quantity $A(t)$ has to be less than $\rho\left(T_{1}-T_{0}\right)-\sigma$ for all $t \in\left[0, T_{1}\right]$ (see Figure 2).

By taking into account the $(\sigma, \rho)$-constraint, we have

$$
A(\tau) \leq \begin{cases}\min \left(\rho \tau, \rho\left(T_{1}-T_{0}\right)-\sigma\right) & \text { if } T_{1} \geq \sigma / \rho, \\ \rho \tau & \text { if } \tau \leq T_{1} \leq \sigma / \rho .\end{cases}
$$

Note that we always have $A(\tau) \leq \sigma+\rho \tau$.

We search for conditions on $T_{0}$ and $T_{1}$ so that the constraints on $A(\tau)$ are as loose as possible. From the first inequality, we have to take $T_{1}-T_{0} \geq \tau+\sigma / \rho$ so that the first inequality is dummy. The second inequality is as loose as possible if $\mathrm{P}\left(T_{1} \geq \tau\right)$ is as small as possible. As we have $\mathrm{P}\left(T_{1} \geq \tau\right)=1-\tau / \mathrm{E}\left(T_{1}-T_{0}\right)$, this quantity is minimal when $\mathrm{E}\left(T_{1}-T_{0}\right)$ is as small as possible. By combining both arguments, we see that $A(\tau)$ is maximal when $\left(T_{1}-T_{0}\right)$ is constant and equal to $\tau+\sigma / \rho$. Under this assumption, we see that $\mathrm{P}(A(\tau) \geq \rho \tau)=\rho \tau /(\sigma+\rho \tau)$. Hence, the arrival process which maximizes $A(\tau)$ is periodic with period $\sigma+\rho / \tau$. In addition, it is quite clear that $A(\tau)$ is maximal when this random variable can take the value $\sigma+\rho \tau$. This is possible only if a burst of size $\sigma$ is followed or preceded by an activity period with length $\tau$. In the first case, we obtain the process $\left(A^{\tau}(t)\right)$ defined above. In the second case, we obtain the arrival process $\left(\hat{A}^{\tau}(t)\right)$ corresponding to a periodic source with period $\tau+\sigma / \rho$, which is silent for a time period with length $\sigma / \rho$, then active for a period with length $\tau$ and finally transmitting a burst of size $\sigma$ before resuming a new period. This process is such that the random variable $\hat{A}^{\tau}(\tau)$ has the same distribution as the random variable $A^{\tau}(\tau)$ given by equation (2.2). Hence, both processes $\left(A^{\tau}(t)\right)$ and $\left(\hat{A}^{\tau}(t)\right)$ achieve the maximal value (in the sense of the strong ordering) of the random variable $A(\tau)$, i.e. the amount of data, which can be transmitted by a $(\sigma, \rho)$-regulated traffic source with jumps of size $\sigma$. 
When $\tau>\sigma / \rho$, we consider the new point process defined as follows: $T_{0}^{\prime}=\inf \left\{T_{m}: T_{m} \geq\right.$ $\tau\}$ and for $n \geq 1, T_{n}^{\prime}=\inf \left\{T_{m}: T_{m}>T_{n-1}^{\prime}+\tau\right\}$. By considering this point process, we can adapt the previous proof in order to show that the process $\left(A^{\tau}(t)\right)$ is such that $A^{\tau}(\tau)$ is an upper bound for $A(\tau)$; the proof is then complete.

The above result allows us to identify the maximal value in the strong ordering sense for the quantity of information, which can be generated by a $(\sigma, \rho)$-regulated arrival process in an arbitrary time interval with length $\tau$. We now turn to the comparison between this maximum value and a Poisson random variable describing the quantity of information generated in an interval with length $\tau$ by a batch Poisson process with rate $\rho / \sigma$ and batch size $\sigma$.

Lemma 3.2. If the arrival process $(A(t))$ has jumps of size $\sigma$, then for all $\tau>0$, we have $A(\tau) \leq_{\text {icx }} B(\tau)$.

Proof. We use the fact that $A(\tau) \leq{ }_{s t} A^{\tau}(\tau)$. Assume first that $\rho \tau \leq \sigma$. From (2.2), simple computations show that

$$
\int_{x}^{\infty} \mathrm{P}\left(A^{\tau}(\tau)>u\right) \mathrm{d} u= \begin{cases}\rho \tau+\frac{x^{2}-4 x \rho \tau}{2(\sigma+\rho \tau)} & \text { for } 0 \leq x \leq \rho \tau, \\ \rho \tau-\frac{2 x \rho \tau+(\rho \tau)^{2}}{2(\sigma+\rho \tau)} & \text { for } \rho \tau \leq x \leq \sigma, \\ \frac{(\rho \tau+\sigma-x)^{2}}{2(\sigma+\rho \tau)} & \text { for } \sigma \leq x \leq \rho \tau+\sigma, \\ 0 & \text { for } x \geq \rho \tau+\sigma .\end{cases}
$$

For the random variable $B(\tau)$ we have, for $x \leq \sigma$,

$$
\int_{x}^{\infty} \mathrm{P}(B(\tau)>u) \mathrm{d} u=\rho \tau-\int_{0}^{x} \mathrm{P}(B(\tau)>u) \mathrm{d} u=\rho \tau-x\left(1-\mathrm{e}^{-\rho \tau / \sigma}\right) .
$$

Using the fact that, for $y \in[0,1]$,

$$
1-\frac{3 y}{2(1+y)} \leq \mathrm{e}^{-y}
$$

we have, for $x \in[0, \rho \tau]$,

$$
\int_{x}^{\infty} \mathrm{P}\left(A^{\tau}(\tau)>u\right) \mathrm{d} u \leq \int_{x}^{\infty} \mathrm{P}(B(\tau)>u) \mathrm{d} u
$$

In addition, for $y \in[0,1]$, as

$$
1-\mathrm{e}^{-y}-\frac{y}{1+y} \leq \frac{y^{2}}{2(1+y)},
$$

it is easily checked that inequality (3.2) holds for $x \in[\rho \tau, \sigma]$.

Finally, for $x$ in the interval $[\sigma, \sigma+\rho \tau]$, we have

$$
\begin{aligned}
\int_{x}^{\infty} \mathrm{P}(B(\tau)>u) \mathrm{d} u & =\rho \tau-\int_{0}^{\sigma} \mathrm{P}(B(\tau)>u) \mathrm{d} u-\int_{\sigma}^{x} \mathrm{P}(B(\tau)>u) \mathrm{d} u \\
& =\rho \tau\left(1-\mathrm{e}^{-\rho \tau / \sigma}\right)-x\left(1-\left(1+\frac{\rho \tau}{\sigma}\right) \mathrm{e}^{-\rho \tau / \sigma}\right) .
\end{aligned}
$$


The function

$$
x \rightarrow x\left(\left(1+\frac{\rho \tau}{\sigma}\right) \mathrm{e}^{-\rho \tau / \sigma}-\frac{x}{2(\sigma+\rho \tau)}\right)
$$

is maximal for $x^{*}=\sigma(1+\rho \tau / \sigma)^{2} \mathrm{e}^{-\rho \tau / \sigma} \in[\sigma, \sigma+\rho \tau]$ and is for all $x \in[\sigma, \sigma+\rho \tau]$ greater than or equal to the minimum of the quantities

$$
\begin{aligned}
& x_{1}=\sigma\left(\left(1+\frac{\rho \tau}{\sigma}\right) \mathrm{e}^{-\rho \tau / \sigma}-\frac{\sigma}{2(\sigma+\rho \tau)}\right), \\
& x_{2}=\sigma\left(1+\frac{\rho \tau}{\sigma}\right)\left(\left(1+\frac{\rho \tau}{\sigma}\right) \mathrm{e}^{-\rho \tau / \sigma}-\frac{1}{2}\right) .
\end{aligned}
$$

These two quantities are greater than or equal to $(\sigma-\rho \tau) / 2+\rho \tau \mathrm{e}^{-\rho \tau / \sigma}$ and, thus, we deduce that (3.2) holds for $x \in[\sigma, \sigma+\rho \tau]$. Combining the above inequalities, we obtain $A^{\tau}(\tau) \leq_{\mathrm{icx}}$ $B(\tau)$, for $\tau \leq \sigma / \rho$.

Let us now consider the case $\rho \tau \geq \sigma$. From (2.3), the random variable $A^{\tau}(\tau)$ can be written as $A^{\tau}(\tau)=\rho \tau-\sigma+A^{\prime}(\tau)$, where the nonnegative random variable $A^{\prime}(\tau)$ is defined by

$$
\mathrm{P}\left(A^{\prime}(\tau)>x\right)= \begin{cases}1-\frac{x}{\sigma+\rho \tau} & \text { for } 0 \leq x \leq \sigma \\ \frac{2 \sigma-x}{\sigma+\rho \tau} & \text { for } \sigma \leq x \leq 2 \sigma \\ 0 & \text { for } x \geq 2 \sigma .\end{cases}
$$

Simple computations then show that

$$
\int_{x}^{\infty} \mathrm{P}\left(A^{\prime}(\tau)>u\right) \mathrm{d} u= \begin{cases}\sigma-x+\frac{x^{2}}{2(\sigma+\rho \tau)} & \text { for } 0 \leq x \leq \sigma, \\ \frac{(2 \sigma-x)^{2}}{2(\sigma+\rho \tau)} & \text { for } \sigma \leq x \leq 2 \sigma \\ 0 & \text { for } x \geq 2 \sigma .\end{cases}
$$

Let $B_{1}$ be the Poisson random variable defined by

$$
\mathrm{P}\left(B_{1}=k \sigma\right)=\frac{1}{k !}\left(\frac{\rho \tau-\sigma}{\sigma}\right)^{k} \exp \left\{-\frac{(\rho \tau-\sigma)}{\sigma}\right\} .
$$

It is easily checked that $(\rho \tau-\sigma)$ seen as a constant random variable is such that $(\rho \tau-\sigma) \leq_{\text {icx }} B_{1}$. As a matter of fact, picking up any convex (not necessarily increasing) function $f$, we have

$$
\begin{aligned}
\mathrm{E}(f(\rho \tau-\sigma))=f(\rho \tau-\sigma) & =f\left(\sum_{k=0}^{\infty} k \sigma \frac{1}{k !}\left(\frac{\rho \tau-\sigma}{\sigma}\right)^{k} \exp \left\{-\frac{(\rho \tau-\sigma)}{\sigma}\right\}\right) \\
& \leq \sum_{k=0}^{\infty} \frac{1}{k !}\left(\frac{\rho \tau-\sigma}{\sigma}\right)^{k} \exp \left\{-\frac{(\rho \tau-\sigma)}{\sigma}\right\} f(k \sigma) \\
& =\mathrm{E}\left(f\left(B_{1}\right)\right) .
\end{aligned}
$$


The random variable $B_{1}$ represents the quantity of information generated by a batch Poisson process with rate $\rho / \sigma$ and batch size $\sigma$ in the time interval $(0, \tau-\sigma / \rho]$.

Let us now consider the Poisson random variable $B_{2}$, independent from $B_{1}$ and defined by

$$
\mathrm{P}\left(B_{2}=k \sigma\right)=\frac{1}{k !} \mathrm{e}^{-1} .
$$

The random variable $B_{2}$ is equal to the quantity of information generated by a batch Poisson process with rate $\rho / \sigma$ and batch size $\sigma$ in the time interval $(0, \sigma / \rho]$.

For $0 \leq x \leq \sigma$, we have

$$
\int_{x}^{\infty} \mathrm{P}\left(B_{2}>u\right) \mathrm{d} u=\sigma-x\left(1-\mathrm{e}^{-1}\right),
$$

and, using the fact that $\rho \tau \geq \sigma$,

$$
\sigma-x+\frac{x^{2}}{2(\sigma+\rho \tau)} \leq \sigma-x+\frac{\sigma}{4} \leq \sigma-x+\sigma \mathrm{e}^{-1} .
$$

It follows that $\int_{x}^{\infty} \mathrm{P}\left(A^{\prime}(\tau)>u\right) \mathrm{d} u \leq \int_{x}^{\infty} \mathrm{P}\left(B_{2}>u\right) \mathrm{d} u$ for $x \in[0, \sigma]$.

For $\sigma \leq x \leq 2 \sigma$, we have

$$
\int_{x}^{\infty} \mathrm{P}\left(B_{2}>u\right) \mathrm{d} u=\sigma\left(1-\mathrm{e}^{-1}\right)-x\left(1-2 \mathrm{e}^{-1}\right),
$$

and

$$
\begin{aligned}
\int_{x}^{\infty} \mathrm{P}\left(A^{\prime}(\tau)>u\right) \mathrm{d} u & \leq \frac{(2 \sigma-x)^{2}}{4 \sigma} \\
& \leq \sigma\left(1-\mathrm{e}^{-1}\right)-x\left(1-2 \mathrm{e}^{-1}\right) \\
& =\int_{x}^{\infty} \mathrm{P}\left(B_{2}>u\right) \mathrm{d} u
\end{aligned}
$$

as the quadratic polynomial $x^{2} / 4-2 x \mathrm{e}^{-1}+\mathrm{e}^{-1}$ is negative for $x \in[1,2]$. Combining the above results, we deduce that $A^{\prime}(\tau) \leq_{\text {icx }} B_{2}$. As $B_{1}+B_{2} \stackrel{\mathrm{D}}{=} B(\tau)$ and $A^{\tau}(\tau)=\rho \tau-\sigma+A^{\prime}(\tau)$, we deduce that $A^{\tau}(\tau) \leq_{\text {icx }} B(\tau)$ and the proof is complete.

The above result compares the random variable $B(\tau)$, describing the quantity of information generated by a batch Poisson process with rate $\rho / \sigma$ and batch size $\sigma$ in an arbitrary time interval of length $\tau$ with the quantity of information $A(\tau)$, which can be generated in the same interval by a $(\sigma, \rho)$-regulated source with jumps of size $\sigma$. However, there exist sources, which are $(\sigma, \rho)$-regulated but with jumps less than $\sigma$. The following result shows that the conclusion of Lemma 3.2 is still valid for this type of source.

Lemma 3.3. Let $\tilde{A}(\tau)$ be the quantity of information, which can be generated in an arbitrary time interval of length $\tau$ by a $(\sigma, \rho)$-regulated source with jumps less than or equal to $\tilde{\sigma}<\sigma$. Then, $\tilde{A}(\tau) \leq_{\mathrm{icx}} A^{\tau}(\tau)$, which entails $\tilde{A}(\tau) \leq_{\mathrm{icx}} B(\tau)$.

Proof. Using exactly the same arguments as in the proof of Lemma 3.1, we can construct the process that maximizes the quantity $\tilde{A}(\tau)$. Thus, we obtain the arrival process $\left(\tilde{A}^{\tau}(t)\right)$, which is periodic with period $\tau+\tilde{\sigma} / \rho$ and which is composed of a burst of size $\tilde{\sigma}$, followed by an activity period of length $\tau$, followed in turn by a silent period of length $\tilde{\sigma} / \rho$ before 
resuming a new period. By replacing $\sigma$ with $\tilde{\sigma}$, the complementary probability distribution function of $\tilde{A}^{\tau}(\tau)$ is given by (2.2) for the case $\rho \tau \leq \tilde{\sigma}$ and (2.3) for the case $\rho \tau \geq \tilde{\sigma}$. From these equations, we immediately check that we do not have $\tilde{A}^{\tau}(\tau) \leq_{s t} A^{\tau}(\tau)$. The proof of the lemma is decomposed into three steps, depending upon the relative positions of the quantities $\sigma, \tilde{\sigma}$ and $\rho \tau$.

Case $1(\rho \tau \leq \tilde{\sigma} \leq \sigma)$. If $\tilde{\sigma}+\rho \tau \leq \sigma$, then it is easily checked that, using (3.1), the inequality

$$
\int_{x}^{\infty} \mathrm{P}\left(\tilde{A}^{\tau}(\tau)>u\right) \mathrm{d} u \leq \int_{x}^{\infty} \mathrm{P}\left(A^{\tau}(\tau)>u\right) \mathrm{d} u
$$

holds for all $x \geq 0$. If $\tilde{\sigma}+\rho \tau \geq \sigma$, the only point to verify is that the above inequality holds for $x \in[\sigma, \tilde{\sigma}+\rho \tau]$. For this purpose, it is sufficient to check that $\tilde{f}(\sigma) \leq f(\sigma)$, where

$$
\tilde{f}(\sigma)=\frac{(\tilde{\sigma}+\rho \tau-\sigma)^{2}}{2(\tilde{\sigma}+\rho \tau)} \quad \text { and } \quad f(\sigma)=\frac{(\sigma+\rho \tau-\sigma)^{2}}{2(\sigma+\rho \tau)} .
$$

As the function $x \rightarrow(x-\sigma)^{2} / x$ is nondecreasing for $x \geq \sigma$, we have $\tilde{f}(\sigma) \leq f(\sigma)$. This entails that (3.3) is valid for all $x \geq 0$.

Case $2(\tilde{\sigma} \leq \rho \tau \leq \sigma)$. We first note, from equation (2.3), that simple computations yield

$$
\int_{x}^{\infty} \mathrm{P}\left(\tilde{A}^{\tau}(\tau)>u\right) \mathrm{d} u= \begin{cases}\rho \tau-x & \text { for } 0 \leq x \leq \rho \tau-\tilde{\sigma}, \\ \frac{\tilde{\sigma}-\rho \tau}{2}+\frac{(2 \rho \tau-x)^{2}}{2(\tilde{\sigma}+\rho \tau)} & \text { for } \rho \tau-\tilde{\sigma} \leq x \leq \rho \tau, \\ \frac{(\rho \tau+\tilde{\sigma}-x)^{2}}{2(\tilde{\sigma}+\rho \tau)} & \text { for } \rho \tau \leq x \leq \rho \tau+\tilde{\sigma}, \\ 0 & \text { for } x \geq \rho \tau+\tilde{\sigma} .\end{cases}
$$

The function $x \rightarrow \rho \tau+\left(x^{2}-4 x \rho \tau\right) /(2(\sigma+\rho \tau))$ is convex and the derivative at $x=0$ is equal to $-2 \rho \tau /(\sigma+\rho \tau) \geq-1$, which implies that, for all $x \geq 0, \rho \tau+\left(x^{2}-4 x \rho \tau\right) /(2(\sigma+$ $\rho \tau)) \geq \rho \tau-x$.

For $x \in[\rho \tau-\tilde{\sigma}, \rho \tau]$, the functions

$$
f_{2}: x \rightarrow \frac{\tilde{\sigma}-\rho \tau}{2}+\frac{(2 \rho \tau-x)^{2}}{2(\tilde{\sigma}+\rho \tau)} \quad \text { and } \quad f_{3}: x \rightarrow \rho \tau+\frac{x^{2}-4 x \rho \tau}{2(\sigma+\rho \tau)}
$$

are decreasing. From the previous arguments, we know that $f_{2}(\rho \tau-\tilde{\sigma}) \leq f_{3}(\rho \tau-\tilde{\sigma})$. Then, for all $x \in[\rho \tau-\tilde{\sigma}, \rho \tau]$, we have $f_{2}(x) \leq f_{3}(x)$ if $\left|f_{2}^{\prime}(x)\right| \geq\left|f_{3}^{\prime}(x)\right|$. Indeed, since the functions are continuous, assuming that there exists an $x_{0}$ such that $f_{3}\left(x_{0}\right)=f_{2}\left(x_{0}\right)$ and an $h$ sufficiently small, $f_{3}\left(x_{0}+h\right)<f_{2}\left(x_{0}+h\right)$. Using the fact that

$$
f_{3}\left(x_{0}+h\right)=f_{3}\left(x_{0}\right)+h f_{3}^{\prime}\left(x_{0}\right)+o(h)<f_{2}\left(x_{0}+h\right)=f_{2}\left(x_{0}\right)+h f_{2}^{\prime}\left(x_{0}\right)+o(h),
$$

we deduce that, by taking sufficiently small $h,\left|f_{3}^{\prime}\left(x_{0}\right)\right|>\left|f_{2}^{\prime}\left(x_{0}\right)\right|$. Simple computations show that

$$
f_{3}^{\prime}(x)=\frac{x-2 \rho \tau}{\sigma+\rho \tau} \quad \text { and } \quad f_{2}^{\prime}(x)=\frac{x-2 \rho \tau}{\tilde{\sigma}+\rho \tau},
$$

and it is clear that $\left|f_{2}^{\prime}(x)\right| \geq\left|f_{3}^{\prime}(x)\right|$ for $x \in[\rho \tau, \rho \tau+\tilde{\sigma}]$, which implies that $f_{3}(x) \geq f_{2}(x)$. 
For $x \in[\rho \tau, \rho \tau+\tilde{\sigma}]$, we have to check that

$$
\frac{(\rho \tau+\tilde{\sigma}-x)^{2}}{2(\tilde{\sigma}+\rho \tau)} \leq \begin{cases}\rho \tau-\frac{2 x \rho \tau+(\rho \tau)^{2}}{2(\sigma+\rho \tau)}, & x \in[\rho \tau, \sigma], \\ \frac{(\rho \tau+\sigma-x)^{2}}{2(\sigma+\rho \tau)}, & x \in[\sigma, \sigma+\rho \tau] .\end{cases}
$$

If $\rho \tau+\tilde{\sigma}<\sigma$, then (3.4) holds because the function on the left-hand side is convex and decreasing from $\tilde{\sigma}^{2} /(2(\sigma+\rho \tau))$, for $x=\rho \tau$, to 0 , for $x=\rho \tau+\tilde{\sigma}$, while the curve of the function on the right-hand side is a straight line, which starts and ends at points with ordinates greater than or equal to these two values.

If $\rho \tau+\tilde{\sigma} \geq \sigma$, we use the same arguments by noting that the value of the left-hand side at point $\sigma$ is equal to $(\rho \tau+\tilde{\sigma}-\sigma)^{2} /(2(\tilde{\sigma}+\rho \tau))=\tilde{f}(\sigma)$ and that of the right-hand side is $(\rho \tau)^{2} /(2(\sigma+\rho \tau))=f(\sigma)$. We already know that $\tilde{f}(\sigma) \leq f(\sigma)$ as $\rho \tau+\tilde{\sigma} \geq \sigma$. For fixed $x \in[\sigma, \tilde{\sigma}+\rho \tau]$, the function $y \rightarrow(y-x)^{2} /(2 y)$ is increasing for $y \geq x$ and we finally deduce that (3.4) is always valid, which in turn implies that (3.3) is valid for all $x \geq 0$.

Case $3(\rho \tau \leq \tilde{\sigma} \leq \sigma)$. We can prove (3.3) by combining the arguments invoked in the previous cases. This ends the proof.

In the above proof, we have considered the worst case that is compatible with jumps of size $\tilde{\sigma}$. But it is also possible to construct a process with jumps of size $\sigma$, which pathwise dominates the process $(\tilde{A}(t))$ so that the conclusion of Lemma 3.2 can be applied to this dominating process and then entails Lemma 3.3. This principle is used to examine the last possibility for the arrival process $(A(t))$ : the case when the arrival process $(A(t))$ has no jumps, which implies that the process $(A(t))$ is continuous.

Lemma 3.4. If the arrival process $(A(t))$ has no jumps, we have $A(\tau) \leq_{\mathrm{icx}} B(\tau)$ for all $\tau>0$.

Proof. If, for all $t, A(t) \leq \rho t$, then we trivially have $A(\tau) \leq_{\mathrm{icx}} B(\tau)$. Indeed, for any increasing convex function $f$, we have

$$
\mathrm{E}(f(A(\tau))) \leq f(\rho \tau) \leq \sum_{n=0}^{\infty} \frac{(\rho \tau / \sigma)^{n}}{n !} \mathrm{e}^{-\rho \tau / \sigma} f(n \sigma)=\mathrm{E}(f(B(\tau))) .
$$

Assume now that the process $(A(\tau))$ is such that there are time periods, when the instantaneous arrival rate (i.e. the right derivative of the function $t \rightarrow A(t)$ ) is greater than $\rho$. We divide the real axis into (random) time intervals $\left(b_{n}, e_{n}\right), n \in \mathbb{Z}$, so that the instantaneous arrival rate is greater than $\rho$ only in these time intervals. We then construct a pathwise dominating process, which has jumps at times $b_{n}$ with magnitude $A\left(b_{n}, e_{n}\right]-\rho\left(e_{n}-b_{n}\right)$ followed by an activity period with length $\left(e_{n}-b_{n}\right)$. The process over the other time intervals is left unchanged. Thus, we construct a new process, which is compatible with the $(\sigma, \rho)$-constraint and pathwise dominates the original process. But for this new process, the conclusions of the previous lemmas hold and the result follows.

Combining the technical Lemmas 3.2, 3.3, and 3.4, we deduce Proposition 3.1. This result shows that for an arbitrary time interval of length $\tau$ and any $(\sigma, \rho)$-regulated arrival process $(A(t))$, the quantity $A(\tau)$ is dominated by $B(\tau)$ in the increasing convex ordering sense. Note that Proposition 3.1 is finer than inequality (1.1) which was proved by [14]. From this inequality, 
we can easily deduce that, for all $\tau \geq 0, A(\tau) \leq_{\text {icx }} B(\tau)+X_{\sigma}$, where the random variable $X_{\sigma}$ is defined by $\mathrm{P}\left(X_{\sigma}= \pm \sigma\right)=\frac{1}{2}$, but we cannot directly obtain $A(\tau) \leq_{\mathrm{icx}} B(\tau)$.

Using the same kind of arguments, we can prove that the conclusion of Proposition 3.1 holds for the corresponding stochastic processes.

Proposition 3.2. If $(A(t))$ is a $(\sigma, \rho)$-regulated arrival process, then $(A(t)) \leq_{\mathrm{icx}}(B(t))$, where $(B(t))$ is a batch Poisson process with rate $\rho / \sigma$ and batch size $\sigma$.

Proof. We prove by mathematical induction that, for all $n \geq 1$ and all $t_{1}<\cdots<t_{n}$, $\left(A\left(t_{1}\right), A\left(t_{2}\right), \ldots, A\left(t_{n}\right)\right) \leq_{\mathrm{icx}}\left(B\left(t_{1}\right), \ldots, B\left(t_{n}\right)\right)$. From Proposition 3.1, we know that the result is true for $n=1$.

Assume that the result is true for $n$ and consider arbitrary $t_{1}<\cdots<t_{n}<t_{n+1}$. We can write

$$
\left(A\left(t_{1}\right), A\left(t_{2}\right), \ldots, A\left(t_{n}\right), A\left(t_{n+1}\right)\right)=\left(A\left(t_{1}\right), A\left(t_{2}\right), \ldots, A\left(t_{n}\right), A\left(t_{n}\right)+A\left(t_{n}, t_{n+1}\right]\right) .
$$

The random variables $A\left(t_{1}\right), A\left(t_{2}\right), \ldots, A\left(t_{n}\right)$ and $A\left(t_{n}, t_{n+1}\right]$ are correlated. However, the arrival process $A(t)$ is also $(\sigma, \rho)$-regulated on the interval $\left[t_{n}, t_{n+1}\right]$ and we know from Lemma 3.1 that the quantity $A\left(t_{n}, t_{n+1}\right.$ ] is maximal in the strong ordering sense for the arrival process $\left(A^{t_{n+1}-t_{n}}(t)\right)$ defined in Section 2, independently of the history of the process for $t \leq t_{n}$ but provided that the point $t_{n}$ is arbitrary. Hence, even though the random variables are correlated, by letting the time origin be arbitrary and by using the induction assumption, we have

$$
\begin{aligned}
& \left(A\left(t_{1}\right), A\left(t_{2}\right), \ldots, A\left(t_{n}\right), A\left(t_{n}\right)+A\left(t_{n}, t_{n+1}\right]\right) \\
& \quad \leq_{\mathrm{icx}}\left(B\left(t_{1}\right), \ldots, B\left(t_{n}\right), B\left(t_{n}\right)+B\left(t_{n+1}-t_{n}\right)\right) .
\end{aligned}
$$

As

$$
\left(B\left(t_{1}\right), \ldots, B\left(t_{n}\right), B\left(t_{n}\right)+B\left(t_{n+1}-t_{n}\right)\right) \stackrel{\mathrm{D}}{=}\left(B\left(t_{1}\right), \ldots, B\left(t_{n}\right), B\left(t_{n+1}\right)\right),
$$

the result follows.

Using the fact that if $X, Y$, and $Z$ are three independent random variables such that $X \leq_{\text {icx }} Y$, then $X+Z \leq_{\text {icx }} Y+Z$, we can easily prove the following result.

Corollary 3.1. Let $N$ be a positive integer and assume that for $i=1, \ldots, N$, the process $\left(A_{i}(t)\right)$ is $\left(\sigma_{i}, \rho_{i}\right)$-regulated. The superposition process $\left(A_{1}(t)+\cdots+A_{N}(t)\right)$ is dominated in the increasing convex ordering sense by the batch Poisson process $(\beta(t))$ with rate $\sum_{i=1}^{N} \rho_{i} / \sigma_{i}$ and random batch size $b$, the distribution of which is given by

$$
\mathrm{P}\left(b=\sigma_{i}\right)=\frac{\rho_{i} / \sigma_{i}}{\sum_{j=1}^{N} \rho_{j} / \sigma_{j}} \text { for } i=1, \ldots, N .
$$

\section{Queueing applications}

In this section we consider a fluid buffer drained at a constant rate $c$ and fed with a $(\sigma, \rho)$-regulated source with stationary increments. Let $A(t)$ denote the quantity of information generated in the time interval $(0, t]$. We assume that $\rho<c$ so that a stationary regime exists for the system. Let $w$ denote the buffer content in the stationary regime. From Reich's formula, we have

$$
w=\sup _{t \geq 0}(A(t)-c t)
$$


As the source is $(\sigma, \rho)$-regulated, we have $A(t) \leq \sigma+\rho t$. Hence, the buffer content $w$ is such that

$$
w=\sup _{t \in[0, T]}(A(t)-c t),
$$

where $T=\sigma /(c-\rho)$, as the buffer is almost surely (a.s.) empty at time $T$.

The objective of this section is to show a stochastic domination property for the buffer content $w$. More precisely, we intend to prove that the random variable $w$ is dominated in the increasing convex ordering sense by the random variable $W$, which is the content in the stationary regime of a buffer drained at a constant rate $c$ and fed with a batch Poisson process with rate $\rho / \sigma$ and batch size $\sigma$. For this purpose, we use the compactness property in some appropriate space of the sample paths of the arrival process $(A(t))$ and we show that for any $\varepsilon>0$, there exists a sequence $t_{1}, \ldots, t_{n}$ such that the supremum in (4.1) satisfies

$$
\sup _{t \in[0, T]}(A(t)-c t) \leq \max _{1 \leq i \leq n}\left(A\left(t_{1}\right)-c t_{1}, \ldots, A\left(t_{n}\right)-c t_{n}\right)+\varepsilon .
$$

Then, using Proposition 3.2, we can prove the desired result.

Before proceeding to the main result of this section, let us introduce some notation and prove preliminary lemmas. Let $D([0, T], \mathbb{R})$ denote the set of càdlàg functions from $[0, T]$ into $\mathbb{R}$ equipped with the Skorokhod topology, i.e. the topology associated with the metric $d$ defined as follows: Let $\Lambda$ denote the class of strictly increasing, continuous mappings of $[0, T]$ onto itself. Note that if $\lambda \in \Lambda, \lambda(0)=0$ and $\lambda(1)=1$. For $x, y \in D([0, T], \mathbb{R})$, define $d(x, y)$ to be the infimum of those positive $\eta$ for which there exists in $\Lambda$ a function $\lambda$ such that

$$
\sup _{t \in[0, T]}|\lambda(t)-t| \leq \eta \quad \text { and } \quad \sup _{t \in[0, T]}|x(t)-y(\lambda(t))| \leq \eta .
$$

The set $D([0, T], \mathbb{R})$ equipped with the Skorokhod topology is a complete and separable space.

To avoid 'pathological' cases, which do not have a physical interpretation, we shall assume in this section that the sample-paths of a $(\sigma, \rho)$-regulated arrival process $(A(t))$ satisfy the following regularity assumptions:

$\left(\mathrm{H}_{1}\right)$ jumps of an arrival process have a minimum size $\Delta>0$ (i.e. if $(A(t))$ has a jump at time $t \geq 0$, then $A(t)-A\left(t^{-}\right) \geq \Delta$ a.s.);

$\left(\mathrm{H}_{2}\right)$ the instantaneous arrival rate cannot exceed a constant $\pi>\rho$ (i.e. for all $t \geq 0, A^{+}(t) \leq$ $\pi$ a.s., where $A^{+}(t)$ is the right derivative of the function $t \rightarrow A(t)$ at point $\left.t\right)$.

The first assumption requires that bursts transmitted by the source contain a minimum amount of information. The last hypothesis corresponds to a peak rate constraint, even though jumps are allowed in the arrival process.

In the following, we assume that assumptions $\left(\mathrm{H}_{1}\right)$ and $\left(\mathrm{H}_{2}\right)$ are implicit and are satisfied by all the $(\sigma, \rho)$-regulated traffic sources. Under these assumptions, the process $(A(t))$ is càdlàg so that the sample paths of the process $(A(t))$ are in the set

$$
\begin{aligned}
\mathcal{A}=\{f \in D([0, T], \mathbb{R}): & f \text { is nondecreasing, has jumps of size greater than } \Delta>0, \\
& \text { for all } t, f^{+}(t) \leq \pi, \text { and, for all } s \leq t, \\
& f(t)-f(s) \leq \min (\rho(t-s)+\sigma)\} .
\end{aligned}
$$

Lemma 4.1. The set $\mathcal{A}$ is a compact subspace of $D([0, T], \mathbb{R})$. 
Proof. Let us first show that the set $\mathcal{A}$ is closed. Let us consider a sequence $\left(x_{n}\right)$ of elements of $\mathcal{A}$ tending to $x \in D([0, T], \mathbb{R})$ for the Skorokhod topology. Let $\varepsilon>0$ and let $x_{n}$ be such that $d(x, y)<\varepsilon$. There exists some $\lambda_{n} \in \Lambda$ such that

$$
\sup _{t \in[0, T]}\left|\lambda_{n}(t)-t\right| \leq \epsilon \quad \text { and } \quad \sup _{t \in[0, T]}\left|x(t)-x_{n}\left(\lambda_{n}(t)\right)\right| \leq \epsilon .
$$

Assume that $s<t$. Then, using the fact that $x_{n}$ is nondecreasing,

$$
x(t) \geq x_{n}\left(\lambda_{n}(t)\right)-\varepsilon \geq x_{n}\left(\lambda_{n}(s)\right)-\varepsilon \geq x_{n}(s)-2 \varepsilon .
$$

As this inequality is valid for all $\varepsilon>0$, we deduce that $x$ is nondecreasing. In addition, for $s \leq t$,

$$
\begin{aligned}
x(t)-x(s) & =x(t)-x_{n}\left(\lambda_{n}(t)\right)+x_{n}\left(\lambda_{n}(t)\right)-x_{n}\left(\lambda_{n}(s)\right)-\left(x(s)-x_{n}\left(\lambda_{n}(s)\right)\right) \\
& \leq 2 \varepsilon+\min \left(\pi\left(\lambda_{n}(t)-\lambda_{n}(s)\right), \rho\left(\lambda_{n}(t)-\lambda_{n}(s)\right)+\sigma\right) \\
& \leq 2(1+\pi) \varepsilon+\min (\pi(t-s), \rho(t-s)+\sigma) .
\end{aligned}
$$

As this inequality is valid for all $\varepsilon>0$, we deduce that, for all $s \leq t, x(t)-x(s) \leq \rho(t-s)+\sigma$. In the same way, we can prove that the jumps of $x$ are greater than or equal to $\Delta$ and that $x^{+}(t) \leq \pi$. Hence, $x \in \mathcal{A}$. This entails that the set $\mathcal{A}$ is closed.

To show that the set $\mathcal{A}$ is compact, we use [2, Theorem 14.3]. We must check that

$$
\sup _{x \in \mathcal{A}} \sup _{t \in[0, T]}|x(t)|<\infty \quad \text { and } \quad \lim _{\delta \rightarrow 0} \sup _{x \in \mathcal{A}} w_{x}^{\prime}(\delta)=0,
$$

where $w_{x}^{\prime}(\delta)=\inf _{\left\{t_{i}\right\}} \max _{0<i \leq r} w_{x}\left[t_{i-1}, t_{i}\right)$, the infimum extending over the finite set $\left\{t_{i}\right\}$ of points such that $0=t_{0}<t_{1}<\cdots<t_{r}=T$ and $t_{i}-t_{i-1}>\delta$ for $i=1, \ldots, r$, and $w_{x}\left[t_{i-1}, t_{i}\right)=\sup _{t_{i-1} \leq s \leq t<t_{i}}|x(t)-x(s)|$.

The first inequality is trivial as, for all $x \in \mathcal{A}, 0 \leq x(t) \leq \rho T+\sigma$. In addition, by assumption $\left(\mathrm{H}_{1}\right)$, the function $x$ has only a finite number of jumps in the time interval $[0, T]$. If $\delta$ is small enough, then one can find a finite set $\left\{t_{i}\right\}$, as above, such that the jumps occur at some points of this set. Then, using the peak rate constraint $\left(\mathrm{H}_{2}\right)$, we have

$$
w_{x}\left[t_{i-1}, t_{i}\right) \leq \pi\left(t_{i}-t_{i-1}\right),
$$

as $x$ is càdlàg and there are no jumps in the time interval $\left[t_{i-1}, t_{i}\right)$. Hence, if $\delta$ is sufficiently small, $w_{x}^{\prime}(\delta) \leq \pi \delta$ and the second condition is satisfied.

Lemma 4.2. Let $\varepsilon>0$. Then, there exists an integer $n>0$ and real numbers $0 \leq t_{1}<t_{2}<$ $\cdots<t_{n} \leq T$ such that, for all $x \in \mathcal{A}$,

$$
\sup _{t \in[0, T]}(x(t)-c t) \leq \max \left(x\left(t_{1}\right)-c t_{1}, x\left(t_{2}\right)-c t_{2}, \ldots, x\left(t_{n}\right)-c t_{n}\right)+\varepsilon .
$$

Proof. Let $\varepsilon>0$. For $x \in \mathcal{A}$, let $\mathcal{B}(x, \varepsilon)=\{y \in \mathcal{A}: d(x, y)<\varepsilon\}$. As $\mathcal{A}$ is compact, there exists an integer $n \geq 1$ and elements $x_{1}, \ldots, x_{n}$ of $\mathcal{A}$ such that $\mathcal{A}$ is covered by the union of the set $\mathscr{B}\left(x_{j}, \varepsilon\right)$ for $j=1, \ldots, n$. Let $x$ be an arbitrary element of $\mathcal{A}$. Then, there exists $1 \leq j \leq n$ such that $x \in \mathscr{B}\left(x_{j}, \varepsilon\right)$. In that case, there exists some $\lambda \in \Lambda$ such that

$$
\sup _{t \in[0, T]}|\lambda(t)-t| \leq \varepsilon \quad \text { and } \sup _{t \in[0, T]}\left|x(t)-x_{j}(\lambda(t))\right| \leq \varepsilon
$$


Hence, for all $t \in[0, T]$,

$$
\begin{aligned}
\left(x_{j}(\lambda(t))-c \lambda(t)\right)-\varepsilon(1+c) & \leq x(t)-c t \\
& =x(t)-x_{j}(\lambda(t))+x_{j}(\lambda(t))-c \lambda(t)+c(\lambda(t)-t) \\
& \leq\left(x_{j}(\lambda(t))-c \lambda(t)\right)+\varepsilon(1+c) .
\end{aligned}
$$

Let $t_{j} \in[0, T]$ such that $\sup _{t \in[0, T]}\left(x_{j}(\lambda(t))-c \lambda(t)\right)=x_{j}\left(t_{j}\right)-c t_{j}$. The above inequality entails that

$$
\begin{aligned}
x_{j}\left(t_{j}\right)-c t_{j} & \leq x\left(t_{j}\right)-c t_{j}+\varepsilon(1+c) \\
& \leq \max \left(x\left(t_{1}\right)-c t_{1}, x\left(t_{2}\right)-c t_{2}, \ldots, x\left(t_{n}\right)-c t_{n}\right)+\varepsilon(1+c),
\end{aligned}
$$

and

$$
\begin{aligned}
& \max \left(x\left(t_{1}\right)-c t_{1}, x\left(t_{2}\right)-c t_{2}, \ldots, x\left(t_{n}\right)-c t_{n}\right) \\
& \quad \leq \sup _{t \in[0, T]}(x(t)-c t) \leq x_{j}\left(t_{j}\right)-c t_{j}+\varepsilon(1+c) .
\end{aligned}
$$

It follows that

$$
\sup _{t \in[0, T]}(x(t)-c t) \leq \max \left(x\left(t_{1}\right)-c t_{1}, x\left(t_{2}\right)-c t_{2}, \ldots, x\left(t_{n}\right)-c t_{n}\right)+2 \varepsilon(1+c) .
$$

By replacing $\varepsilon$ with $\varepsilon /(2(1+c))$ and by relabeling, if necessary, the $t_{j}$ in order to get an increasing sequence, we obtain the desired result.

Theorem 4.1. Let $(A(t))$ be the arrival process of a $(\sigma, \rho)$-regulated source with stationary increments such that $\rho<c$. Let $(C(t))$ be a nondecreasing process with stationary increments such that $\mathrm{E}(C(t)) \leq \gamma t$ with $\gamma<c$. Consider two fluid queues drained with a constant rate $c$. The first one is fed with the input process $(A(t))$ and the buffer content in the stationary regime is denoted by $w=\sup _{t \geq 0}(A(t)-c t)$. The second queue is fed with the input process $(C(t))$ and the buffer content in the stationary regime is denoted by $W=\sup _{t \geq 0}(C(t)-c t)$. If $(A(t)) \leq_{\text {icx }}(C(t))$, then $\mathrm{E}(w) \leq \mathrm{E}(W)$.

Proof. Let $\varepsilon>0$. As the source $(A(t))$ is $(\sigma, \rho)$-regulated, we have

$$
w=\sup _{t \in[0, T]}(A(t)-c t)
$$

with $T=\sigma /(c-\rho)$ and the sample paths of $(A(t))$ are in the set $\mathcal{A}$. From Lemma 4.2, there exists an integer $n>0$ and points $t_{1}<t_{2}<\cdots<t_{n}$ such that

$$
\sup _{t \in[0, T]}(A(t)-c t) \leq \varepsilon+\max \left(A\left(t_{1}\right)-c t_{1}, A\left(t_{2}\right)-c t_{2}, \ldots, A\left(t_{n}\right)-c t_{n}\right) \quad \text { a.s. }
$$

This entails that

$$
\mathrm{E}(w) \leq \varepsilon+\mathrm{E}\left(\max \left(A\left(t_{1}\right)-c t_{1}, A\left(t_{2}\right)-c t_{2}, \ldots, A\left(t_{n}\right)-c t_{n}\right)\right) .
$$

By assumption, $(A(t)) \leq_{\mathrm{icx}}(C(t))$ and as the function $f: \mathbb{R}^{n} \rightarrow \mathbb{R}$ defined by

$$
f\left(x_{1}, x_{2}, \ldots, x_{n}\right)=\max \left(x_{1}-c t_{1}, x_{2}-c t_{2}, \ldots, x_{n}-c t_{n}\right)
$$


is convex increasing, we have

$$
\begin{aligned}
\mathrm{E}(\max & \left.\left(A\left(t_{1}\right)-c t_{1}, A\left(t_{2}\right)-c t_{2}, \ldots, A\left(t_{n}\right)-c t_{n}\right)\right) \\
& \leq \mathrm{E}\left(\max \left(C\left(t_{1}\right)-c t_{1}, C\left(t_{2}\right)-c t_{2}, \ldots, C\left(t_{n}\right)-c t_{n}\right)\right) \\
& \leq \mathrm{E}\left(\sup _{t \geq 0}(C(t)-c t)\right) \\
& =\mathrm{E}(W) .
\end{aligned}
$$

We deduce that, for all $\varepsilon>0, \mathrm{E}(w) \leq \varepsilon+\mathrm{E}(W)$ and the result follows.

Using Corollary 3.1 and the fact that the superposition of regulated sources is also regulated, we can easily deduce the following result.

Corollary 4.1. Assume that, for $i=1, \ldots, N,\left(A_{i}(t)\right)$ is the arrival process of a $\left(\sigma_{i}, \rho_{i}\right)$ regulated source with stationary increments and assume that $\sum_{i=1}^{N} \rho_{i}<c$. Consider a fluid queue fed with the superposition of those regulated sources and let $w$ denote the buffer content in the stationary regime; $w=\sup _{t \geq 0}\left(\sum_{i}^{N} A_{i}(t)-c t\right)$. Then, $\mathrm{E}(w) \leq \mathrm{E}(W)$, where $W$ is the content in the stationary regime of a fluid queue fed with the arrival process $(\beta(t))$ defined in Corollary 3.1.

The quantity $\mathrm{E}(W)$ can be computed easily using the Pollaczek-Khinchin formula and is given by

$$
\mathrm{E}(W)=\frac{1}{2(c-\rho)} \sum_{i=1}^{N} \rho_{i} \sigma_{i} .
$$

It is worth noting that the stochastic bound obtained in this paper is weaker than that derived in [9] in the single input case. Indeed, in that paper, it was shown that $w \leq_{s t} W$, when the buffer is fed with a single $(\sigma, \rho)$-regulated arrival process. The same result, however, seems to be rather difficult to show in the multiple input case. Nevertheless, in the many sources asymptotics setting, it is shown in [16] that the result is asymptotically true.

\section{References}

[1] Baccelli, F. ANd Brémaud, P. (1994). Elements of Queueing Theory (Appl. Math. 26). Springer, New York.

[2] Billingsley, P. (1968). Convergence of Probability Measures. John Wiley, New York.

[3] Chang, C.-S. (2000). Performance Guarantees in Communication Networks. Springer, London.

[4] Chang, C.-S., Song, W. and Chiu, Y.-M. (2001). On the performance of multiplexing independent regulated inputs. In Proc. 2001 ACM Sigmetrics, ACM Press, New York, pp. 184-193.

[5] Cruz, R. L. (1991). A calculus for network delay. I. Network elements in isolation. IEEE Trans. Inf. Theory 37, $114-131$.

[6] Cruz, R. L. (1991). A calculus for network delay. II. Network analysis. IEEE Trans. Inf. Theory 37, $132-141$.

[7] FIDLER, M. (2004). Elements of probabilistic network calculus applying moment generating functions. Tech. Rep. 12, Institut Mittag Leffler.

[8] Firoiu, V., Le Boudec, J.-Y., Towsley, D. and Zhang, Z. L. (2002). Theories and models for internet quality of service. Proc. IEEE, pp. 1565-1591.

[9] Guillemin, F., Likhanov, N., Mazumdar, R. and Rosenberg, C. (2003). Buffer overflow bounds for multiplexed regulated traffic streams. In Proc. 18th Intenat. Teletraffic Congress 2003, eds J. Charzinsky et al. Elsevier, pp. 491-500.

[10] Guillemin, F. M., Likhanov, N., Mazumdar, R. R. and Rosenberg, C. P. (2002). Extremal traffic and bounds for the mean delay of multiplexed regulated traffic streams. In Proc. IEEE INFOCOM 2002, IEEE, pp. 985-993.

[11] Kesidis, G. And Konstantopoulos, T. (2000). Extremal traffic and worst-case performance for queues with shaped arrivals. In Analysis of Communication Networks: Call Centres, Traffic and Performance, American Mathematical Society, Providence, RI, pp. 159-178. 
[12] Le Boudec, J.-Y. and Thiran, P. (2001). Network Calculus. Springer, Berlin.

[13] Massoulié, L. (1998). Large deviation ordering of point processes in some queueing networks. Queueing Systems 4, 317-335.

[14] Massoulié, L. and Busson, A. (2000). Stochastic majorization of aggregates of leaky bucket-constrained traffic streams. Preprint, Microsoft Research, Cambridge.

[15] TURNER, J. (1986). New directions in communications (or which way in the information age?). IEEE Commun. Mag. 24, 8-15.

[16] Ying, Y., Guillemin, F., Mazumdar, R. and Rosenberg, C. (2005). Buffer overflow asymptotics for multiplexed regulated traffic. To appear in Performance Evaluation. 